Article original

\title{
Intérêt zootechnique de l'utilisation de la pulpe de betterave ensilée dans l'alimentation du porc lourd
}

\author{
Giovanna Martelli*, Paolo Parisini, Luca Sardi, Rosanna Scipioni, \\ Giorgio Vignola, Andrea Panciroli, Archimede Mordenti
}

Dipartimento di Morfofisiologia veterinaria e Produzioni animali, Université de Bologne,

Via Tolara di Sopra, 50, 40064 Ozzano Emilia, Bologne, Italie

(Reçu le 17 avril 1998 ; accepté le 28 octobre 1998)

\begin{abstract}
The effects of sugar beet pulp silage in the diets for heavy pigs. Sixty Landrace $\times$ Large White pigs ( 30 females and 30 castrated males), ranging in live weight from $35-160 \mathrm{~kg}$, were homogeneously divided into three groups fed as follows: $\mathrm{T}$, control group, in which animals received a 'traditional' diet based on cereals and soybean meal; T2, in which plain pressed beet pulp silage (PPBPS) partially replaced barley (for $15 \%$ on a dry matter basis); and T3, in which molassed pressed beet pulp silage (MPBPS) substituted barley at the same rate as in group T2. The animals were fed at the rate of $9 \%$ of their metabolic live weight up to a maximum of $3.2 \mathrm{~kg} \cdot \mathrm{pig}^{-1} \mathrm{~d}^{-1}$. Pigs were individually weighed at $0,35,105$ and $182 \mathrm{~d}$ of trial. Feed intake was recorded to calculate the feed conversion rate (FCR). Pigs on the PPBPS diet showed a significant $(P<0.05)$ improvement of daily weight gain. No differences $(P>0.05)$ concerning FCR were observed between groups, although energy conversion was improved for pigs receiving both types of the by-product. Pigs were slaughtered at around $160 \mathrm{~kg}$ live weight. The main qualitative parameters of carcasses, meat $(\mathrm{pH}$ and colour) and fat (fatty acid composition) were collected. Pigs receiving pressed beet pulp showed a significant $(P<0.05)$ improvement of the weight of the gastrointestinal tract, inducing a slight reduction of the dressing out percentage in comparison with control animals. Back fat thickness and muscle percentage did not differ depending on the diet; these parameters were significantly $(P<0.01)$ affected only by the sex of the animals. Similarly, the yield of the lean and fatty cuts was not affected by the diets, the significant differences $(P<0.01)$ being exclusively related to the different hormonal status of the entire females and castrated males. Meat colour and $\mathrm{pH}$ were not affected by the diet as well as ham fat acidic composition. Raw hams were cured over a 13-month period. No differences between the groups were observed with regard to ham weight loss. Our data indicate that pressed beet pulp silage, either molassed or plain, can be profitably used in the diets for heavy pigs. The high energy supply of beet pulp, which is related to its non-starch fermentable polysaccharides content, can induce a reduction of feed cost due to cereals such as barley sparing. (C) Elsevier / Inra)
\end{abstract}

pig / beet pulp silage / growth / carcass quality / ham

* Correspondance et tirés à part.

E-mail : Parisini@vet.unibo.it 
Résumé - L'étude porte sur l'influence de la substitution partielle (15\% de la MS du régime) de l'orge par un pourcentage identique de matière sèche fournie par la pulpe de betterave surpressée ensilée ou bien la pulpe de betterave mélassée ensilée sur les performances de croissance-finition du porc lourd (poids à l'abattage $160 \mathrm{~kg}$ environ). La réponse de croissance a été globalement et significativement $(p<0,05)$ en faveur du groupe qui recevait la pulpe non mélassée. À l'abattage, les animaux ayant reçu l'aliment sans pulpe ont eu un rendement de carcasse légèrement supérieur en raison d'un poids inférieur $(p<0,05)$ du tube digestif plein et vidé. Les animaux alimentés avec le régime contenant la pulpe mélassée ont présenté une épaisseur du lard dorsal légèrement supérieure. Les carcasses des femelles étaient significativement moins grasses avec un pourcentage de muscle plus élevé ainsi qu'un pourcentage de pièces maigres supérieur $(p<0,01)$. Aucune différence relative aux paramètres de qualité ( $\mathrm{pH}$, couleur de la viande et composition en acides gras) n'a été relevée entre les différents régimes. Il en va de même pour les données relatives au poids des jambons et aux pertes de poids relevées au moment des phases les plus importantes de la production. (@) Elsevier / Inra)

porc / pulpe de betterave ensilée / croissance / qualité carcasse / jambon

\section{INTRODUCTION}

L'emploi de résidus de l'industrie agroalimentaire dans l'alimentation porcine est une pratique qui a toujours accompagné l'élevage de cette espèce en Italie [34]. En particulier, le binôme élevage porcin/ résidus de l'industrie fromagère (lactosérum en l'occurrence) a longtemps été considéré comme essentiel pour une bonne réussite économique de ce type d'élevage, et aujourd'hui encore les règlements pour la production des jambons typiques de «Parma » et de « San Daniele » incluent cette spécificité.

De nos jours, l'emploi dans l'alimentation animale de matières qui seraient autrement détruites trouve sa justification pour des raisons d'ordre non seulement économique mais aussi social et de sauvegarde de l'environnement. Cette utilisation ne doit comporter aucun risque pour la santé de l'animal et du consommateur, et permettre d'obtenir de bonnes performances d'élevage et une qualité élevée des carcasses et des produits dérivés. C'est donc dans cette optique que l'emploi de la pulpe de betterave a trouvé sa raison d'être, non seulement dans l'alimentation des ruminants mais aussi dans celle du porc, espèce dont les capacités de digestion d'aliments fibreux ont été clairement mises en évidence au cours de ces dernières années [11].

La conservation par ensilage de la pulpe de betterave surpressée est une technique assez récente qui a l'avantage de réduire les coûts par rapport à la déshydratation, tout en obtenant un produit dont les qualités nutritives et la digestibilité sont bien préservées, voire améliorées.

À ce jour, les travaux sur l'emploi de la pulpe de betterave ensilée ont déjà mis en évidence les effets de ce produit dans l'alimentation du porc. En particulier l'addition de fibres en provenance de la pulpe de betterave ensilée a permis de constater une certaine réduction des pathologies gastriques [22] ainsi que, du point de vue comportemental, une diminution sensible des agressions chez les truies en gestation [11]. Dans cette étude spécifique aucune réduction des performances de reproduction n'a été notée. En revanche, la consommation d'eau, le volume d'urine produit et le taux d'urée des urines ont été réduits de même que la quantité de phosphore fécal et urinaire. Lors d'un éssai de digestibilité [29] effectué sur des porcs qui recevaient des quantités croissantes (de 0 à $24 \%$ de la MS de la ration) de pulpe de betterave ensilée, une améliora- 
tion du bilan azoté a entraîné une réduction substantielle de l'excrétion d'urée dans les urines. Ces résultats seraient donc à considérer avec attention en vue d'une réduction de la pollution azotée provoquée par les lisiers porcins [19].

En ce qui concerne les performances d'élevage, l'emploi de régimes riches en fibres en période de croissance-finition provoque normalement une réduction de la croissance, comme conséquence de la réduction de la teneur en énergie digestible et, surtout, en énergie nette de la ration avec toutefois une variabilité très dépendante des caractéristiques des parois végétales.

Le travail présenté ici s'intègre donc dans le cadre des recherches sur les possibilités d'utilisation de la pulpe de betterave dans l'alimentation du porc. En particulier l'objectif a été d'étudier l'influence de la substitution partielle d'une céréale, l'orge en l'occurrence, par un pourcentage identique de pulpe de betterave surpressée ensilée ou bien de pulpe de betterave mélassée ensilée. Les performances d'élevage et la composition corporelle à l'abattage du porc lourd (poids à l'abattage $160 \mathrm{~kg}$, environ), ainsi que les caractéristiques des jambons produits ont été considérés.

\section{MATÉRIEL ET MÉTHODES}

Pour la réalisation de cet essai, 60 porcs Landrace $\times$ Large White, mâles castrés et femelles en effectif équivalent ont été utilisés. Les animaux ont été répartis de façon homogène en fonction du poids et du sexe en trois groupes (T1, T2, T3) correspondant aux trois régimes alimentaires à tester. Les 20 porcs de chaque groupe étaient logés dans quatre box comprenant cinq animaux du même sexe, situés dans un bâtiment à ambiance contrôlée. L'essai prévoyait trois phases (de 35 à $60 \mathrm{~kg}$, de 60 à $100 \mathrm{~kg}$ et de 100 à $160 \mathrm{~kg}$ ) durant lesquelles ont été testés les trois régimes suivants :

- les animaux du groupe T1 (contrôle) recevaient un aliment traditionnel formulé pour les phases de croissance-finition en accord avec les recommandations prévues par l'Inra [12] ;
- les animaux des autres groupes recevaient le même aliment dans lequel $15 \%$ de la MS de la farine d'orge étaient remplacés respectivement par une quantité identique de MS fournie par de la pulpe de betterave surpressée ensilée (T2) et surpressée ensilée et mélassée (T3) ; le pourcentage de mélasse était de $5 \%$ du volume total de la pulpe fraîche.

La composition des régimes expérimentaux est présentée dans le tableau I et les données analytiques dans le tableau II. Ces différents aliments étaient offerts à raison de $9 \%$ du poids vif métabolique (p.v. ${ }^{0,75}$ ), avec des augmentations graduelles jusqu'à un maximum de $3,2 \mathrm{~kg}$ par animal et par jour. La distribution était sous forme liquide suivant un rapport matière sèche/eau de $1 / 4$. À cet effet, un système automatique de distribution a été employé. Il prévoyait le mélange en quantité contrôlée des différents aliments auxquels était ajoutée la pulpe de betterave. La distribution aux différents box a été faite deux fois par jour.

Afin de calculer le gain de poids et l'indice de consommation, les animaux ont été pesés individuellement à $0,35,105$ et $182 \mathrm{j}$ d'élevage. L'essai strict d'élevage (enregistrement des poids vifs et calcul des indices de consommation) s'est terminé à cette dernière date, quand au moins un tiers des animaux avait atteint le poids d'abattage $(160 \mathrm{~kg})$. L'effectif resté alors en élevage a naturellement continué à recevoir les régimes expérimentaux jusqu'au départ à l'abattoir.

À l'abattage, effectué après un jeûne de $24 \mathrm{~h}$, le poids de la carcasse chaude, du foie et des organes digestifs pleins et vidés, comprenant l'appareil génital chez les femelles, a été mesuré sur dix porcs de chaque groupe.

On a ensuite procédé, selon les Directives Européennes [4] et avec un appareil optique FatO-Meater (FOM, SFK, Copenhague, DK), aux mesures de l'épaisseur de la bardière (points $\mathrm{X}_{2}$ et $\mathrm{X}_{4}$ ) et du muscle Latissimus dorsi (point $\mathrm{X}_{5}$ ); les mesures ont servi à calculer le pourcentage de muscle de la carcasse. L'épaisseur de la bardière a été déterminée avec le système Intrascope au niveau du point $\mathrm{X}_{2}$, situé entre la $3^{\mathrm{e}}$ et la $4^{\mathrm{e}}$ côte, à $8 \mathrm{~cm}$ de la ligne moyenne de fente de la carcasse. Une détermination du $\mathrm{pH}$ au moyen d'un pH-mètre portatif a été effectuée à 45 min et à $24 \mathrm{~h}$ de l'abattage au niveau des muscles Latissimus dorsi et Semimembranosus.

La découpe de la demi carcasse droite a été réalisée $24 \mathrm{~h}$ après l'abattage ; les pièces maigres (jambon, longe et épaule) et grasses (panne, bardière, poitrine et gorge) ainsi obtenues ont permis 


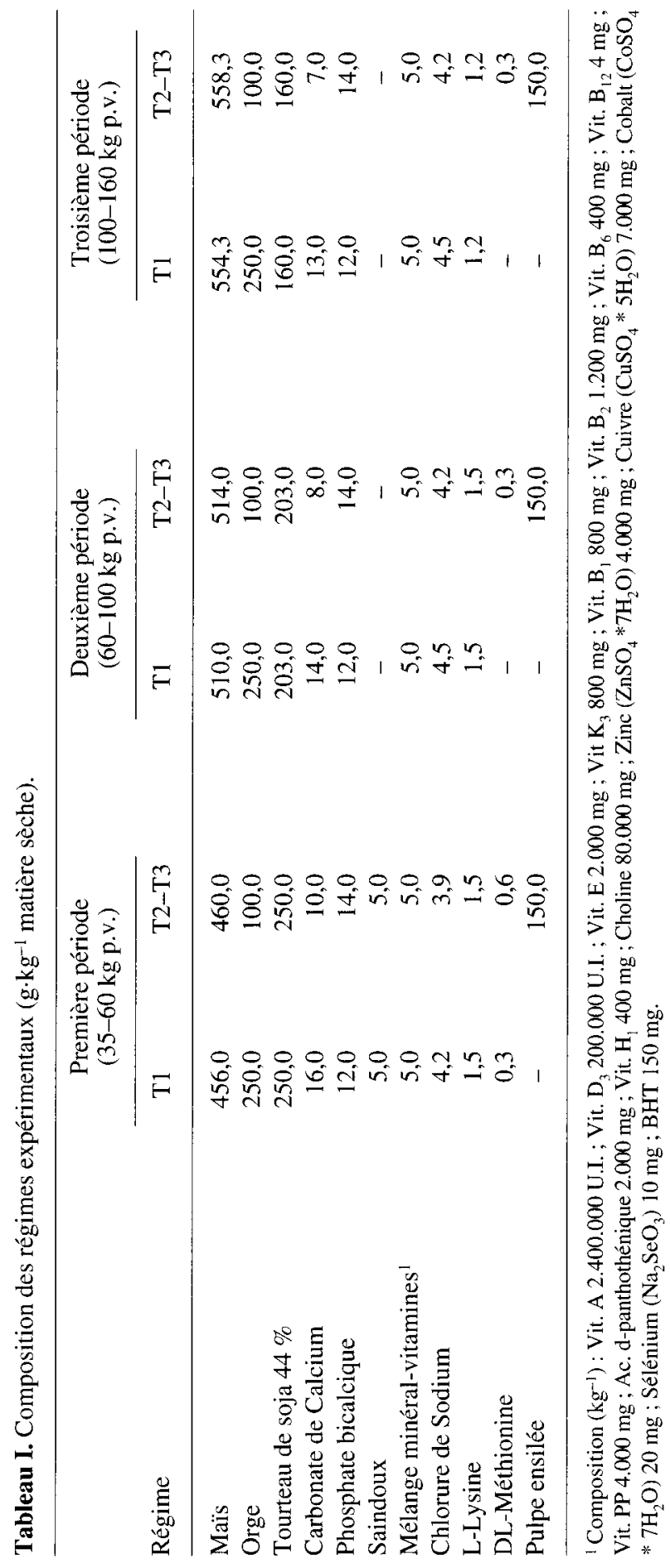




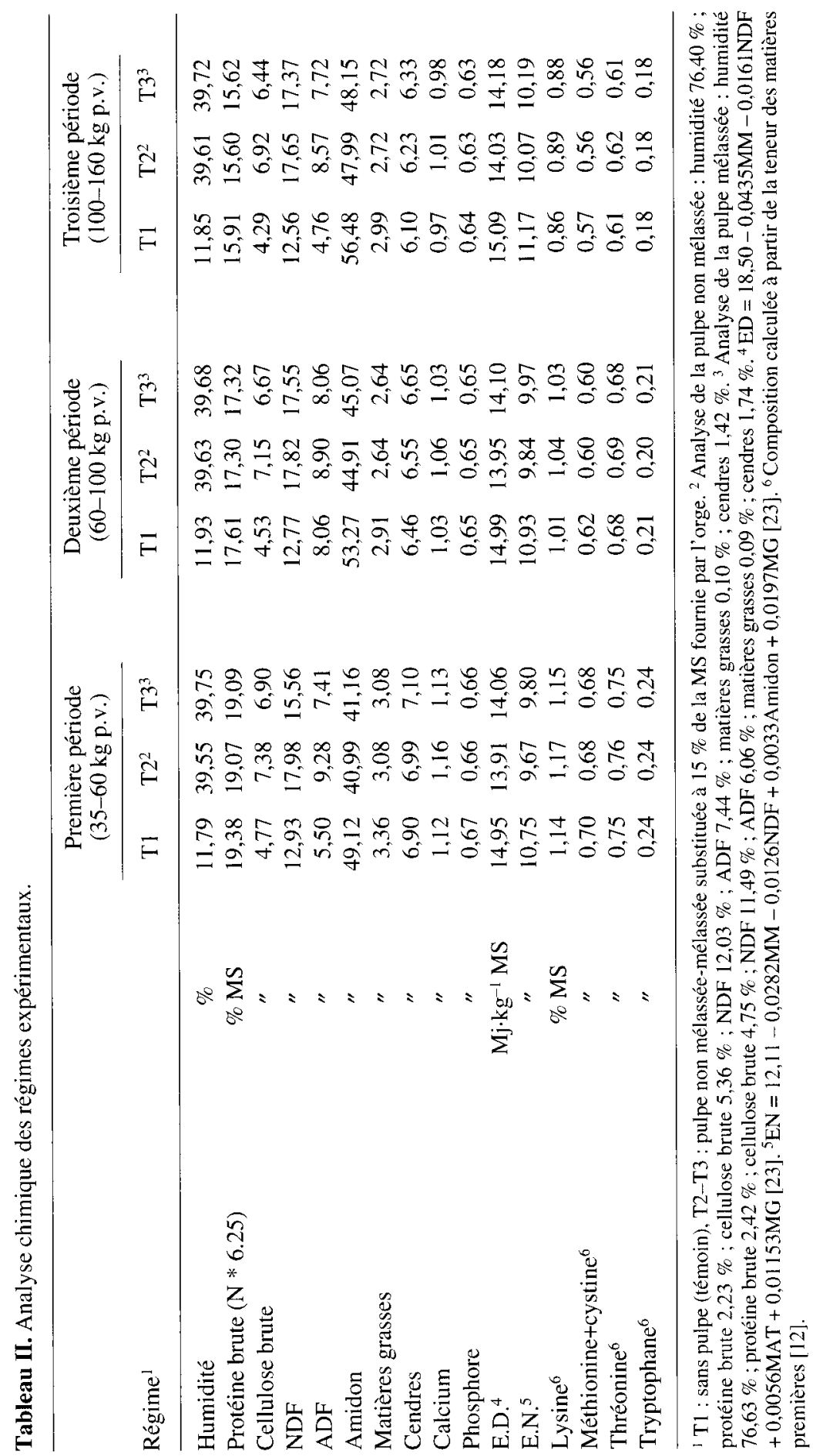


de calculer leur pourcentage par rapport à la demi carcasse ainsi que le rapport pièces maigres/ pièces grasses. Enfin la couleur de la viande au niveau des muscles Semimembranosus et Biceps femoris du jambon (après l'élimination de la graisse en excès) a été mesurée selon le système Hunter L.a.b. au moyen d'un colorimètre portatif Minolta Chroma meter CR200.

Au laboratoire, la composition en acides gras de la couche adipeuse du jambon a été déterminée par chromatographie en phase gazeuse ; ceci a permis de calculer le coefficient d'insaturation [20].

Les analyses classiques (teneur en matière sèche, cendres, protéine brute, amidon, fibre brute) des différents régimes et des deux types de pulpe ont aussi été effectuées selon les méthodes courantes [1]. Les fractions NDF et ADF ont été déterminées selon la méthode de Van Soest et al. [32]. La teneur en lysine et acides aminés soufrés totaux a été calculée à partir de la teneur moyenne des matières premières incorporées dans les régimes. La teneur en énergie digestible et nette des aliments a été calculée à partir des équations de régression proposées par Noblet et al. [23] (tableau II).

Enfin, la maturation (effectuée selon les normes prévues pour le jambon de Parme) de 48 jambons, répartis de façon équivalente selon le sexe de l'animal et le type d'aliment distribué pendant la période de croissance-finition a été suivie pendant 13 mois. La production des jambons typiques italiens prévoit différentes phases de salaison (qui durent, au total, de trois à quatre semaines), de repos (75-85 j) et de maturation (neuf mois), qui se déroulent en conditions de température et hygrométrie contrôlées. Les pertes de poids des jambons ont été relevées après chacune de ces différentes phases.

Toutes les données ont été traitées statistiquement par analyse de la variance (ANOVA) en utilisant le logiciel SPSS/PC+ [30]. Pour les différentes variables mesurées, les effets retenus ont été les régimes et le sexe des animaux. L'interaction sexe $\times$ régime a été retirée du modèle car clle n'était pas significative. Le modèle mathématique à effets fixés retenu s'écrit :

$$
X_{i j k}=\mu+\alpha_{i}+\beta_{j}+\varepsilon_{i j k}
$$

avec $X_{i j k}=$ mesure faite d'un paramètre pour le $k^{e}$ animal recevant le $i^{c}$ régime appartenant au $j^{e}$ sexe $; \mu=$ moyenne générale du paramètre obscrvé ; $\alpha_{i}=$ effet du régime ( $\mathrm{i}=1$ à 3 ) ; $\beta_{j}=$ effet du sexe $(j=1$ à 2$) ; \varepsilon_{i j k}=$ erreur expérimentale.

\section{RÉSULTATS}

\subsection{Performances zootechniques}

\subsection{Croissance et durée de l'élevage}

La réponse de croissance (tableau III) a été globalement et significativement $(p<0,05)$ en faveur du groupe qui recevait la pulpe non mélassée (T2). En revanche, aucune différence n'a été observée entre la croissance des mâles castrés et celle des femelles : celles-ci, partant d'un poids vif légèrement supérieur ont réalisé un gain moyen journalier sensiblement identique aux mâles.

La durée d'engraissement effective, c'està-dire jusqu'au poids final d'abattage d'environ $160 \mathrm{~kg}$, a été inférieure de $10 \mathrm{j}$ pour les animaux alimentés avec la pulpe non mélassée : conséquence directe du gain de poids plus élevé par rapport aux autres régimes. Cette différence n'a toutefois pas été significative dans l'analyse statistique.

\subsubsection{Consommation alimentaire}

L'ingestion journalière d'aliment qui, rappelons le, a été rationnée pendant toute la phase d'élevage, a été identique pour tous les régimes et dans toutes les phases d'élevage (tableau IV). Ceci a conduit, dans la première période d'élevage ( 1 à $35 \mathrm{j}$ ), à une certaine amélioration non significative de l'indice de consommation alimentaire en faveur des animaux recevant la pulpe ensilée non mélassée.

Durant cette même période, l'indice de consommation exprimé en énergie a mis en évidence de façon significative $(p<0,05)$ la bonne utilisation de l'aliment par les animaux dont les régimes contenaient la pulpe ensilée. Cette tendance s'est confirmée pendant la totalité de la durée de l'essai, mais de façon non significative. 


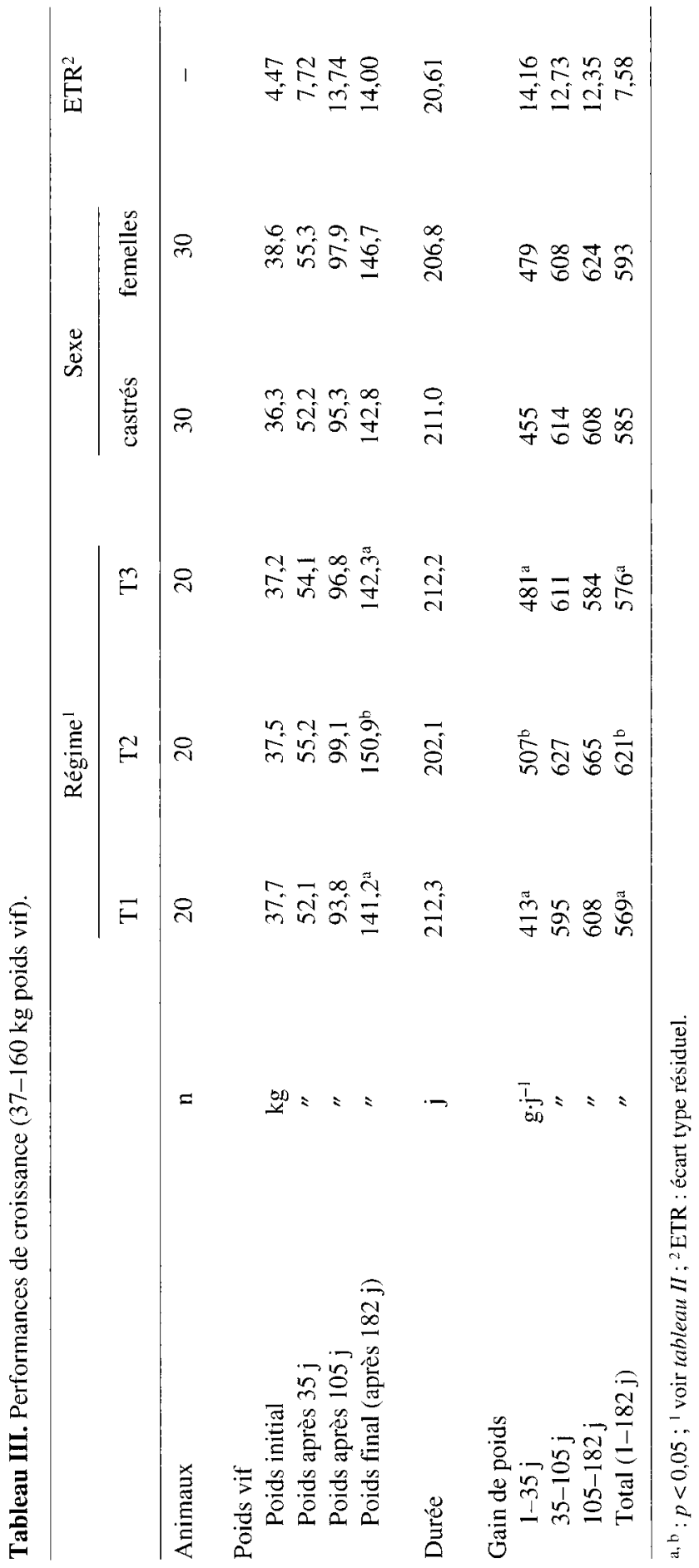




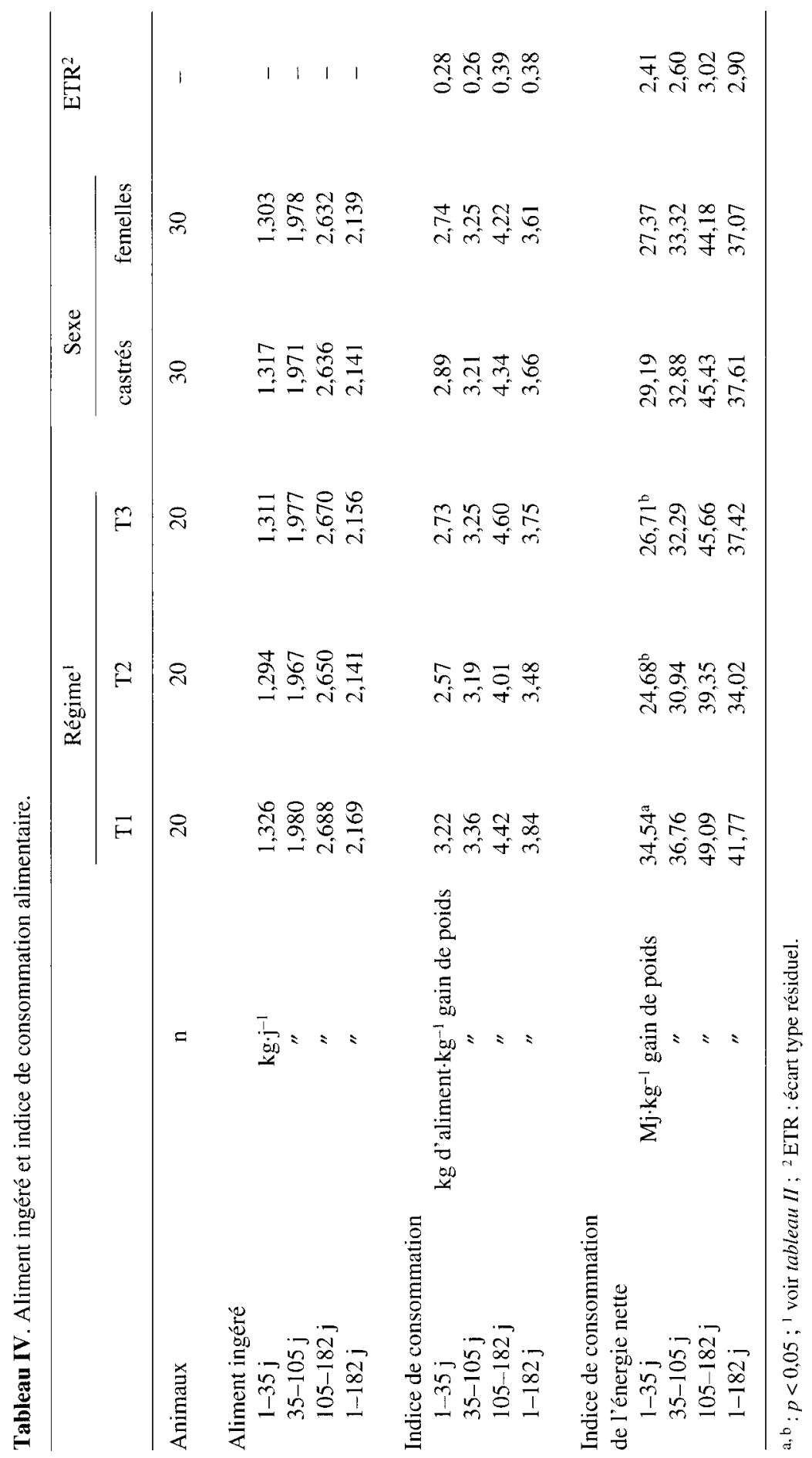




\subsection{Caractéristiques de la carcasse}

À l'abattage (tableau $V$ ), le poids de la carcasse chaude et le rendement en carcasse ne différaient pas de manière significative entre les différents régimes. Les animaux ayant reçu l'aliment sans pulpe présentaient un rendement légèrement supérieur à ceux des deux autres groupes, en raison d'un poids nettement inférieur $(p<0,05)$ du tube digestif plein (estomac, intestin grêle et gros intestin, et appareil génital chez les femelles), du tube digestif vide et du contenu viscéral. Le rendement en carcasse a été en faveur des mâles castrés par suite de l'incidence du poids des viscères, notamment de l'appareil génital chez les femelles.

L'épaisseur du lard dorsal mesurée avec l'appareil Intrascope a été légèrement supérieure chez les animaux alimentés avec le régime contenant la pulpe mélassée. Cela s'est traduit par une réduction d'environ un point de pourcentage de muscle de la carcasse par rapport aux animaux recevant les deux autres régimes. L'effet du sexe sur ces deux paramètres a été très significatif $(p<0,01)$; les femelles, comme dans la plupart des cas, présentaient une carcasse moins grasse et un pourcentage de muscle plus élevé.

\subsection{Découpe}

À la découpe (tableau VI), le poids de la demi carcasse, ainsi que le pourcentage des différentes pièces, ont été semblables entre les différents régimes. Toutefois les données relatives aux animaux alimentés avec la pulpe mélassée ont confirmé la tendance à l'accumulation de graisse déjà observée aux mesures Intrascope et FOM.

De même, les femelles ont présenté un pourcentage de pièces maigres (notamment de jambon et de longe) significativement supérieur aux mâles castrés $(p<0,01)$. La bardière était en revanche plus lourde chez les mâles $(p<0,01)$.

\subsection{Qualité de la viande et maturation des jambons}

Nous n'avons pas constaté de différences significatives entre les animaux recevant les différents régimes en ce qui concerne les paramètres de qualité mesurés par le $\mathrm{pH}$, la couleur de la viande (tableau VII) et la composition en acides gras d'échantillons de graisse prélevés au niveau du jambon (tableau VIII). En ce qui concerne ce dernier paramètre, il faut souligner la tendance (même si les différences ne sont pas significatives) à la réduction des acides gras insaturés ainsi que de l'indice d'insaturation chez les animaux qui recevaient la pulpe, en particulier lorsque celle-ci est mélassée.

Le poids des jambons et les pertes de poids en pourcentage relevés au moment des phases les plus importantes de la production n'ont pas mis en évidence de différences substantielles entre les différents régimes (tableau $I X$ ). Par ailleurs, on a constaté une diminution de la perte de poids pour les jambons provenant des mâles castrés $(p<0,05)$ en raison du degré plus élevé d'adiposité de ceux-ci.

\section{DISCUSSION}

L'ensemble des données enregistrées permet avant tout d'affirmer que l'emploi alimentaire d'un sous-produit tel que la pulpe de betterave conservée par ensilage ne détériore pas les performances du porc élevé jusqu'à $160 \mathrm{~kg}$. L'ensilage, s'il est bien réalisé s'avère donc une méthode de conservation économique permettant d'obtenir un aliment apprécié par le porc.

\subsection{Effets sur les performances zootechniques}

Le gain moyen quotidien des animaux recevant les régimes à base de pulpe non mélassée a été plus élevé que celui des animaux alimentés de façon traditionnelle, ce 

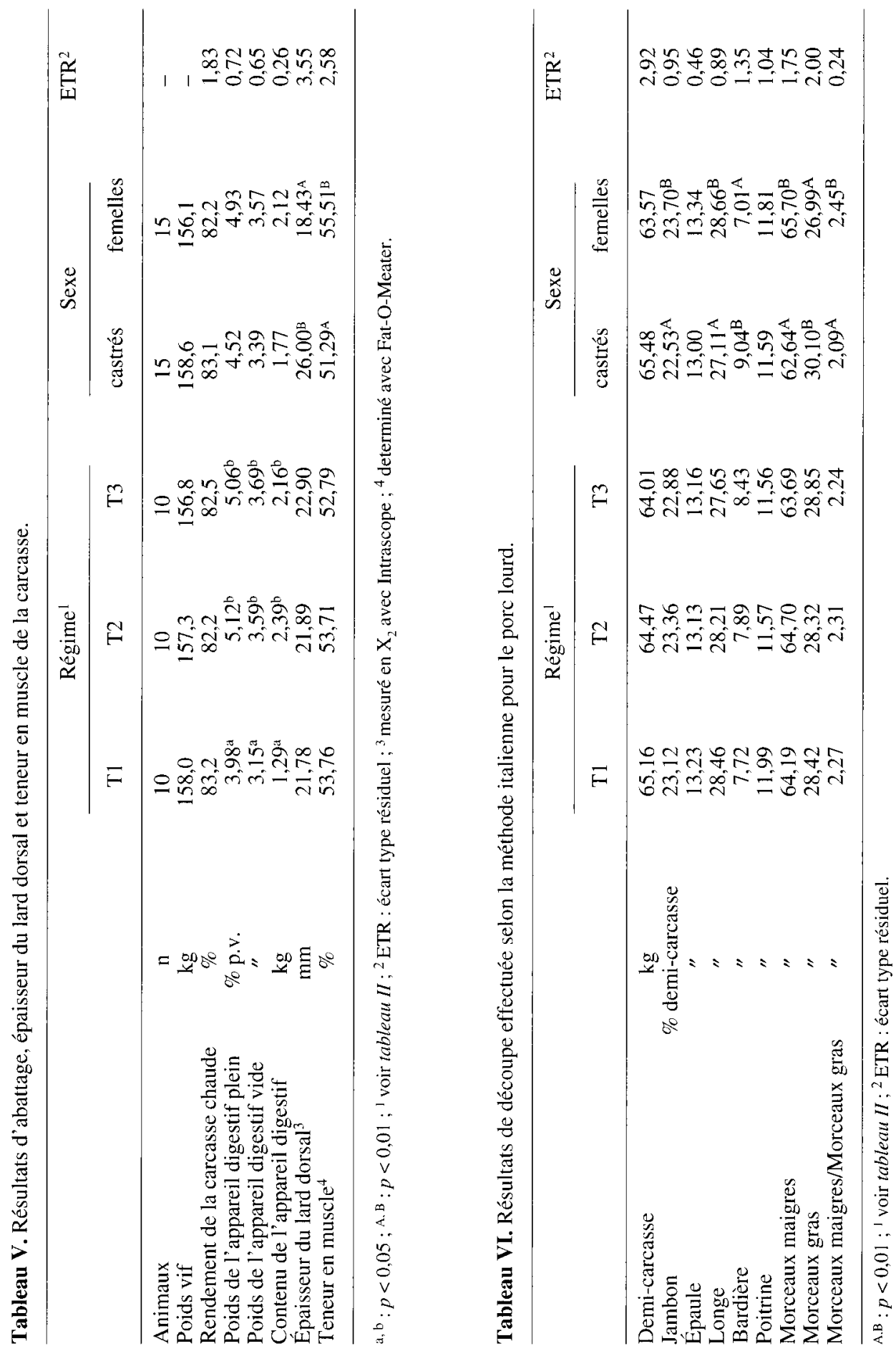


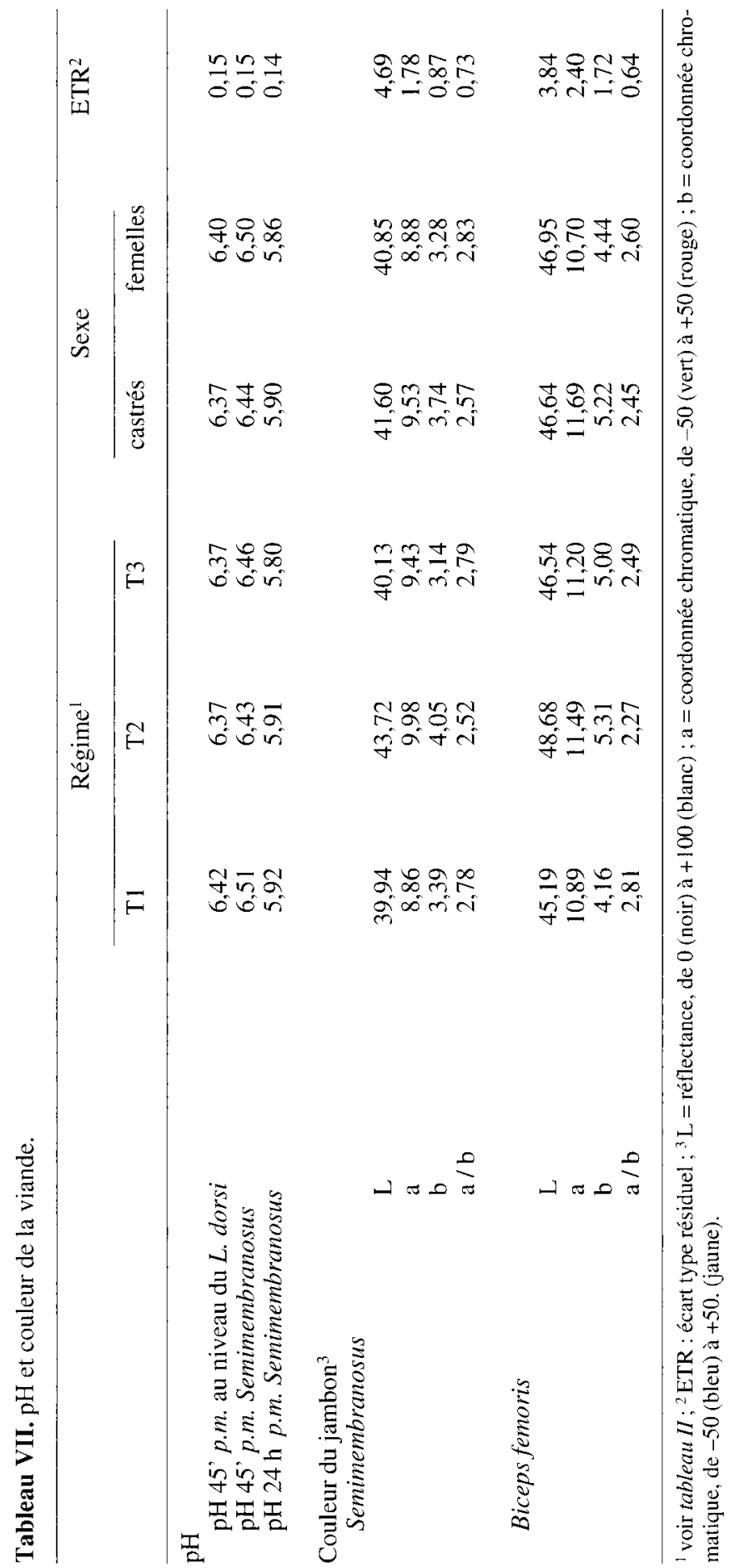




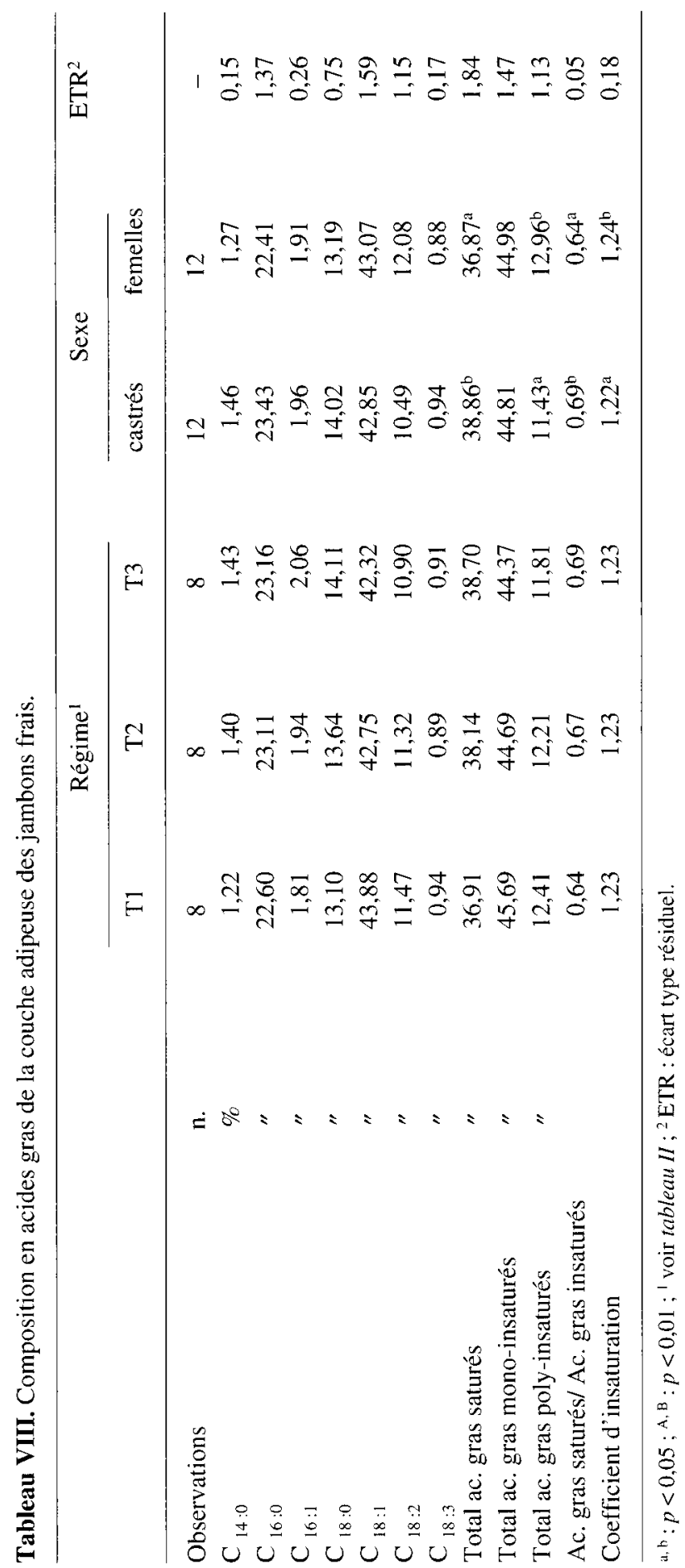




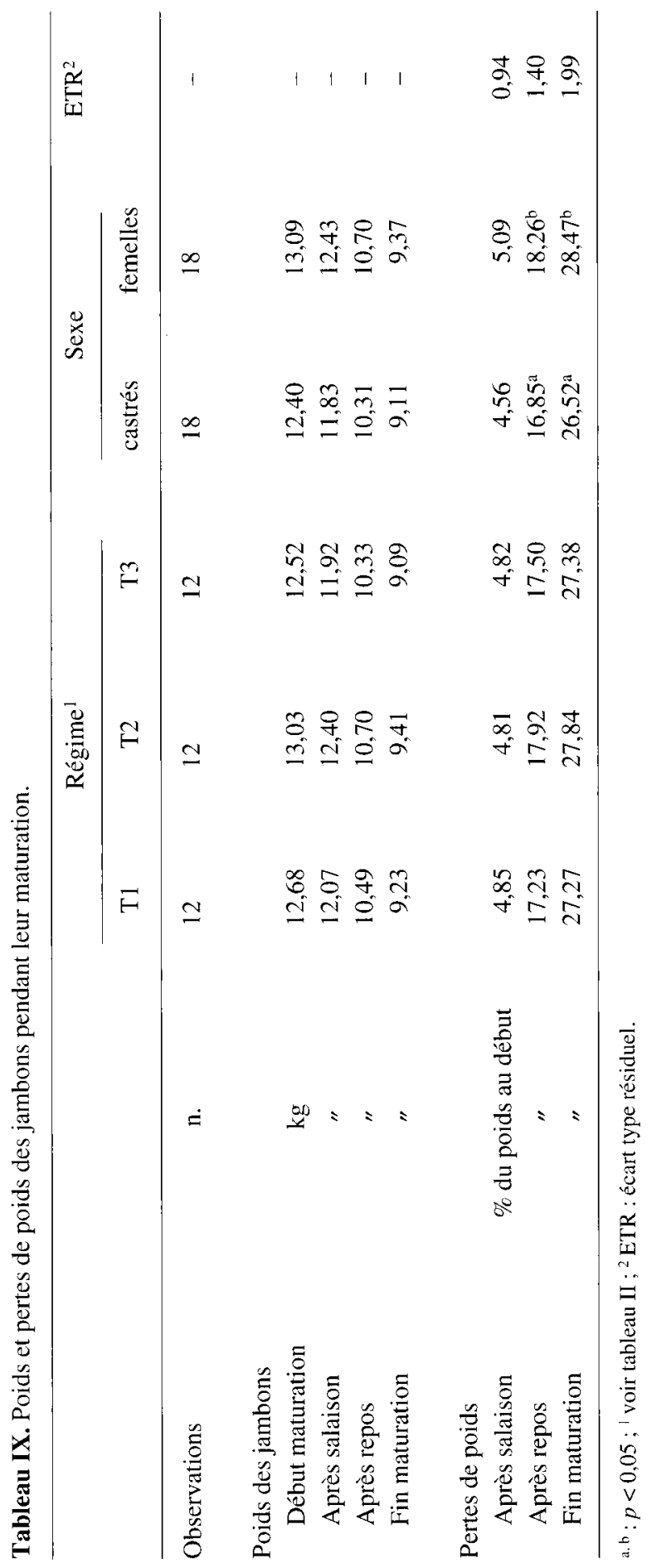


qui s'est traduit par une réduction globale de la durée d'élevage. Si l'on tient compte du fait que la quantité d'aliment ingéré était sensiblement identique pour les trois régimes, on peut en déduire que la réduction apparente de l'apport d'énergie due à l'incorporation de la pulpe n'a pas influencé de façon négative la croissance. Cette donnée pourrait trouver sa justification dans la bonne utilisation des polysaccharides non amylacés (PNA) par le porc en croissancefinition. Plusieurs chercheurs ont en effet montré que les PNA subissent déjà une dégradation partielle au niveau de l'intestin grêle $[3,13]$ mais qu'ils sont surtout utilisés sous forme d'AGV (acides gras volatils) au niveau du gros intestin, grâce à une importante population bactérienne et à un temps de séjour important de l'aliment $[6,8$, 10]. Ainsi, la réduction théorique de la teneur en énergie des régimes riches en fibre devrait probablement être revue en tenant compte de l'utilisation possible de certains polysaccharides, particulièrement fermentescibles et présents en grande quantité dans des aliments comme la pulpe de betterave [26]. À cet égard Haaskma [11] a proposé de reconsidérer l'évaluation énergétique des aliment riches en PNA. Les données de digestibilité mesurées directement sur le porcelet [17] ainsi que sur le porc en croissance [9] semblent confirmer une bonne utilisation des constituants pariétaux de la pulpe pour la production d'AGV. Cela semble d'autant plus vrai chez le porc adulte [7] recevant ce type d'aliment à long terme [2], en favorisant un bon développement de la flore bactérienne. Ainsi s'expliqueraient non seulement l'absence d'effets négatifs des régimes contenant la pulpe [16] mais aussi la tendance à l'amélioration des performances de croissance constatée chez le porc comme chez le porcelet.

L'adjonction de mélasse ne semble pas influencer de façon positive les performances d'élevage qui ont été globalement inférieures à celles des animaux recevant la pulpe ensilée seule et semblables à celles observées avec le régime traditionnel. Cela pourrait être dû à une utilisation moins efficace de l'aliment, peut être liée à la présence d'environ $1 \%$ en plus de cendres brutes dans la pulpe mélassée et à l'augmentation de la vitesse de transit des ingesta. On peut par ailleurs souligner que le porc lourd, comme la truie, est capable de valoriser un régime riche en PNA, car sa capacité digestive vis-à-vis de ces substances est supérieure à celle du porcelet et du porc de $100 \mathrm{~kg} \mathrm{[7,} \mathrm{24,} \mathrm{25].}$

\subsection{Effets sur les caractéristiques de la carcasse}

Les animaux recevant les régimes contenant la pulpe ont présenté un rendement légèrement inférieur, en raison du poids nettement plus élevé de leurs organes digestifs pleins. Ce résultat confirme ceux de Zhu et al. [33] et doit être attribué au poids plus élevé du contenu intestinal conséquence d'une rétention d'eau plus élevée [14, 16, 18] ainsi que d'une augmentation du poids des organes digestifs eux mêmes $[21,28]$.

La présence d'un pourcentage élevé de fibre fermentescible peut aussi entraîner une augmentation de la profondeur des cryptes du cæcum et, par conséquence, améliorer la capacité d'absorption des acides gras volatils qui, à ce niveau, représentent les produits les plus importants de la fermentation des glucides, notamment des pectines [21].

L'introduction de la pulpe non mélassée n'a pas déterminé, contrairement à ce qu'ont observé Kay et al. [15] et Lizardo et al. [16], une réduction significative de l'épaisseur de la bardière ni une augmentation conséquente du pourcentage de muscle de la carcasse. Au contraire, les animaux recevant la pulpe mélassée ont eu tendance à déposer plus de graisse et ont présenté un pourcentage en muscle inférieur à celui des animaux recevant les autres régimes. Les données relatives au rapport pièces maigres/pièces grasses reflètent cette tendance. Toutes les mesures effectuées sur les carcasses se 
situent toutefois parfaitement dans la gamme de variations normale pour le porc lourd. Les différences de réponse à l'emploi de la pulpe par rapport aux porcins élevés dans d'autres pays pourraient être attribuées aux différentes durées d'élevage ainsi qu'à l'origine génétique des animaux. Thielmans et Bodart [31] avaient déjà constaté que l'emploi de régimes dans lesquels $18 \%$ de la MS étaient constitués de pulpe ensilée provoquait un ralentissement de la vitesse de croissance, sans toutefois entraîner des modifications importantes au niveau de la carcasse des animaux. Nous avions déjà obtenu des performances semblables au groupe de contrôle chez des pores qui recevaient de la pulpe de betterave ensilée à raison de $17 \%$ de la MS de la ration [27] ; les caractéristiques des carcasses et de la viande de ces animaux ne différant pas de celles de porcs alimentés de façon traditionnelle.

Il est intéressant de remarquer que l'emploi des deux types de pulpe n'a provoqué aucune modification du coefficient d'insaturation du gras du jambon.

Bien plus évidentes les différences de caractéristiques liées à l'effet du sexe ; les femelles sont, en accord avec les résultats de Lizardo et al. [16], toujours plus maigres que les mâles, avec une proportion de jambon et de longe nettement plus élevée. Ce résultat, à mettre en relation avec les différences hormonales entre les deux sexes [5], s'est traduit par une réduction significative de la perte de poids à la maturation pour les jambons provenant des sujets mâles castrés dont l'épaisse couverture en graisse provoque une réduction substantielle de la perte d'eau.

\section{CONCLUSIONS}

La substitution de $15 \%$ de la MS de la farine d'orge par une quantité équivalente de pulpe de betterave ensilée ne pénalise en aucun cas les performances zootechniques et les caractéristiques de la carcasse du porc lourd. Ceci entraîne une économie substan- tielle d'aliment chez le porc qui doit être conduit à un poids élevé. L'addition de mélasse détermine des performances globalement moins favorables et, par conséquent, ne représente pas un avantage. Cet essai confirme enfin les capacités du porc à utiliser favorablement certains aliments dont le contenu en polysaccharides non amylacés (type pectines) est élevé.

\section{REMERCIEMENTS}

Les auteurs remercient Eridania SpA (Gênes, Italie) pour le soutien financier, ainsi que V. Balduini, M. Dall Olio et Mlle Paola Parazza pour leur collaboration technique.

\section{RÉFÉRENCES}

11] AOAC, Official Methods of Analysis, 15th edn., Washington DC, 1990.

121 Anugwa F.O., Varel V.H., Dickson J.S., Pond W.G., Krook L.P., Effects of dietary fibre and protein concentration on growth, feed efficiency, visceral organs weight and large intestine population in the gastrointestinal tract of pigs, J. Nutr. 119 (1989) 879-886.

1.3] Bach Kuudsen K.E., Jensen B.B., Hansen I., Digestion of polysaccharides and other major components in the small and large intestine of pigs fed diets consisting of oat fractions rich in beta-glucan, Br. J. Nutr. 70 (1993) 537-556.

141 CEE Décision de la Commission 89/50, J. Off. CEE n. L 20 du 25/1/1989, 27-30.

15| Clatus R., Weiler U., Endocrine regulation of growth and metabolism in the pig: a review, Livest. Prod. Sci. 37 (1994) 245-260.

[6] Fadel J., Newman R.K., Newman C.W., Graham $H$., Effects of baking hulless barley on the digestibility of dietary components as measured at the ileum and in the feces of pigs, J. Nutr. 119 (1989) 722-726.

17] Fernandey J., Jorgensen J., Digestibility and absorption of nutrients as affected by libre content in the diet of the pig. Quintitative aspects, Livest. Prod. Sci. 15 (1986) 53-71.

[8] Graham H.. Hesselman K., Jonsson E., Åman P., Influence of beta-glucanase supplementation on digestion of a barley-based diet in the pig gastrointestinal tract, Nutr. Repts. Int. 34 (1986) 1089-1096.

191 Graham H., Hesselman K., Åman P., The influence of wheat bran and sugar beet pulp on the digestibility of dietary components in a cereal based pig diet, J. Nutr. 116 (1986) 242-251. 
|l0| Graham H., Fadel J.G., Newman C.W., Newmam R.K., Effect of pelleting and beta-glucanase supplementation on the ileal and fecal digestibility of a barley-based diet in the pig, J. Anim. Sci. 67 (1989) 386-402.

[1 II Haaksma J., Sugar heet pulp in livestock nutrition. A review, Institute for Rational Sugar Production (IRS), Bergen op Zoom (NL), 1994.

[12] Inra, L'alimentation des animaux monogastriques : porc, lapin, volailles, Inra, Paris, 1989.

[13] Jensen B.B., Jorgensen H., Effect of dietary fibre on microbial activity and microbial gas production in various regions of the gastrointestinal tract of pigs, Appl. Env. Micr. 60 (1994) 1897-1904.

[14] Just A., The influence of crude fibre from cereals on the net energy value for growth in pigs, Livest. Prod. Sci. 9 (1982) 569-580.

[15] Kay R.M., Simmins P.H., Harland J.L., The use of molassed sugar-beet feed in growing pig diets and the effect of inclusion rates on subsequent performance, Anim. Prod. 50 (1990) 154 (Abstr).

[16] Lizardo R., Peiniau J., Lebreton Y., Aumaitre A., Effets de l'incorporation de pulpe de betterave dans les aliments du porcelet et du porc en croissance : performances de croissance, digestibilité et composition corporelle, Ann. Zootech. 46 (1997) 281-294.

[17] Longland A.C., Carruthers J., Low A.G., The ability of piglets 4 to 8 weeks old to digest and perform on diets containing 2 contrasting sources of non-starch polysaccharide, Anim. Prod. 58 (1994) 405-410.

[18] Low A.G., Secretory response of the pig gut to non starch polysaccharides, Anim. Feed Sci. Technol. 23 (1989) 55-65.

[19] Manini R., Piva A., Prandini A., Mordenti A., Piva G., Dourmad J.Y., Protein retention in Italian heavy pigs: development of a factorial approach for the determination of lysine requirement. Livest. Prod. Sci. 47 (1997) 253-259.

[20| Mourot J., Peiniau P., Mounier A., Effets de l'acide linoléique alimentaire sur l'activité des enzymes de la lipogenèse dans les tissus adipeux chez le porc, Reprod. Nutr. Dev. 34 ( 1994) 213-220.

121] Martelli G., Barazzoni A.M., Lalatta Costerbosa G., Sardi L., Parisini P., Anatomical traits of the gastrointestinal tract of pigs fed pressed beet pulp silage, in: LI Congrès National S.I.S.Vet., Bologna (I) 17-20 septembre 1997, pp. $477-478$.

[22| Muttinelli F., Formigoni A., Use of ensiled forages in heavy pig nutrition. Note III : Effect on gastric pathology. in : XLVII Congrès National S.I.S.Vet., Mantova (I) 29 septembre- I $^{\text {er }}$ octobre 1988, pp. 1203-1205.
[23] Noblet J., Fortune H., Dubois S., Henry Y.. Nouvelles bases d'estimation des teneurs en énergie digestible, métabolisable et nette des aliments pour le porc, Inra, Paris, 1989.

[24] Nohlet J., Shi X.S., Comparative digestibility of energy and nutrients in growing pigs fed ad libitum and adults sows fed at maintenance, Livest. Prod. Sci. 34 (1993) 137-152.

[25] Noblet J., Shi X.S., Effect of body weight on digestive utilisation of energy and nutrients of ingredients and diets in pigs, Livest. Prod. Sci. 37 ( 1994$)$ 323-338.

[26] Noblet J.. Bach Knudsen K.E., Comparative digestibility of wheat, maize and sugar beet pulp non-starch polysaccharides in adult sows and growing pigs, in: VIIth Int. Symp. on Digestive Physiology in Pigs, Saint Malo (F), 26-28 mai 1997, Ed. J.P. Laplace, C. Fevrier, A. Barbeau, EAAP publ, no. 88, Inra, Paris, 1997 pp. 57I-574.

[27] Parisini P., Sardi L., Mordenti A., Martelli G., Panciroli A., Polpe secche e polpe surpressate insilate nell'alimentazione del suino pesante, Riv.Suinicoltura 32 (1991) 47-52.

[28] Rundgren M., Growing pig performance : effects of dietary fibre, the halothane gene, transportation and mixing. Doctoral Thesis, Sveriges Lantbruksuniversitet, Inst. f. husdjurens utfodring och vard, Rapport no. 172. 1988.

[29] Scipioni R., Martelli G., Marchetti S., Parisini P., Piva A., Nitrogen balance in pigs fed with different amounts of pressed beet pulp silage (PBPS), in: Int. Congr. Nitrogen Flow in Pig Production and Environmental Consequences, Wageningen (NL), 8-1] juin 1993, Ed. M.W.A. Verstegen, L.A. Den Hartog. G.J.M. van Kempen. J.H.M. Metz. EAAP Publ. no. 69, Pudoc Scientific Publishers, Wageningen 1993. pp. 195-199.

[30] SPSS/PC+ V.3 Base Manual, SPSS Inc., Chicago, IL, USA. 1988.

[31] Thielmans M.F., Bodart C., Les pulpes surpressées ensilées de betterave sucrière dans l'alimentation du porc à l'engrais. Revue de l'Agriculture, 36 (1983) 1411-1414.

132] Van Soest P.J., Robertson J.B., Lewis B.A. Methods for dietary fiber, neutral detergent fiber, and nonstarch polysaccharides in relation to animal nutrition, J. Dairy Sci. 74 (1991) 3583-3597.

[33] Zhu J.Q., Fowler V.R., Fuller M.F., Digestion of ummolassed sugar-beet pulp in young growing pigs and implications for the growth supporting values of fermented energy, Anim. Prod. 50 (1990) 531-539.

[34] Zicarelli L., Piccolo V., Nizza A., Intrieri F. Perrucci G., Indagine sui coefficienti di digeribilità apparente in alcune popolazioni suine meridionali prevalentemente alimentate con sottoprodotti agro-industriali diffusi nel mezzogiorno, Zoot. Nutr. Anim. 5 (1979) 103-109. 